\title{
El papel de los márgenes extensivo e intensivo en el crecimiento de las exportaciones manufactureras españolas por sectores tecnológicos*
}

\author{
Juan A. Máñez \\ Francisco Requena-Silvente \\ María E. Rochina-Barrachina \\ Juan A. Sanchis-Llopis \\ Universitat de València y ERICES
}

\section{Resumen}

En este trabajo se realiza una descomposición del crecimiento de las exportaciones de manufacturas españolas en sus márgenes extensivo e intensivo. A su vez, cada margen está formado por dos componentes: las nuevas relaciones de comercio y su valor (para el margen extensivo); y, las transacciones comerciales que sobreviven y su cambio de valor (para el margen intensivo). Para ello, utilizamos la metodología propuesta por Besedeš y Prusa (2011) y comparamos la evolución del sector exportador español con el de Alemania, utilizando datos de exportaciones bilaterales de productos manufacturados obtenidos de Naciones Unidas (COMTRADE), durante el periodo 1991-2005. Nuestro interés se centra en examinar las diferencias en la evolución exportadora de los sectores manufactureros diferenciados según su contenido tecnológico (sectores de tecnología baja, media-baja, mediaalta y alta). Los resultados muestran que la contribución de cada componente al crecimiento de las exportaciones varía de manera importante por sectores tecnológicos así como entre ambos países para un mismo sector tecnológico. Además, la comparación de España con Alemania, a través de ejercicios contra-factuales, indica que el componente de supervivencia es el elemento más débil en el crecimiento de las exportaciones españolas, especialmente en el sector de alta tecnología, y que los componentes del margen extensivo son, en general, el punto fuerte de las exportaciones españolas.

Palabras clave: exportaciones, sectores tecnológicos, margen extensivo, margen intensivo.

Clasificación JEL: F1.

\section{Abstract}

We analyse the growth of exports in Spanish manufacturing by technological sectors through its decomposition into extensive and intensive margins. These margins are decomposed into four distinct components: new export relationships and their value (for the extensive margin); and, having export relationships survive or persist and deepening existing relationships (for the intensive margin). For that purpose we use and extend the methodology developed by Besedes and Prusa (2007). Further, we compare the Spanish manufacturing performance in terms of these margins and by technological sector with

* Los autores agradecen la financiación recibida del Ministerio de Ciencia e Inovación (proyectos ECO2008-04576/ECON, ECO2008-06395-C05-03 y ECO2008-04059/ECON) y de la Generalitat Valenciana (programas PROMETEO/098 y PROMETEO/068). También agradecemos a Asier Minondo por habernos facilitado los datos de comercio utilizados en este trabajo. Una versión de este trabajo ha sido publicada previamente como papel de trabajo n. ${ }^{\circ} 653$ de la Colección de Documentos de Trabajo de la Fundación de las Cajas de Ahorros (FUNCAS). 
the behaviour of Germany. We conduct ourstudy using disaggregated bilateral manufacturing exports for the two countries involved in theanalysis with the rest of the world. These data come from the UN Commodity Trade StatisticsDatabase (COMTRADE) for the period 1991-2005. Our results show first that the relativeimportance of each component differs by technological sector, independently of the consideredcountry, and also between countries for the same technological sector. Further, the comparison of Spain with Germany, using counterfactual exercises, reveals that the surviving components is the weakest element in explaining Spanish exports growth, specially for the high-tech sector and that the components of the extensive margins are, in general, the strongest component in Spanish exports.

Keywords: exports, technological sector, extensive margins, intensive margins.

JEL classification: $F 1$.

\section{Introducción}

Existe un amplio número de trabajos que analiza el crecimiento a largo plazo del comercio desde la óptica de la contribución de los márgenes (intensivo y extensivo) de comercio y sus determinantes ${ }^{1}$. En particular, el margen extensivo de exportación refleja el cambio en el valor de las exportaciones como resultado de la creación de nuevas relaciones de comercio, mientras que el margen intensivo se refiere al cambio en el valor de las exportaciones de las relaciones de comercio que sobreviven o perduran. La mayoría de estudios encuentra que los dos márgenes son igual de importantes para explicar el crecimiento de las exportaciones en el largo plazo (más de diez años), pero cuando se acorta el periodo de análisis el margen extensivo pierde rápidamente importancia a favor del margen intensivo.

Recientemente, Besedeš y Prusa (2011) han identificado dos componentes diferentes dentro del margen intensivo: la «supervivencia» de las relaciones de comercio existentes y la «profundización» del valor de comercio de las relaciones que sobreviven. Estos autores demuestran que la «supervivencia» es tan importante como la «profundización» a la hora de explicar la evolución de las exportaciones de un país en el largo plazo. Además, el papel protagonista de la «supervivencia» exportadora explica el escaso peso del margen extensivo como determinante del crecimiento de las exportaciones a corto plazo, debido a las altísimas tasas de mortalidad entre las nuevas relaciones comerciales frente a las que ya están consolidadas ${ }^{2}$.

En este trabajo se realiza una descomposición del crecimiento de las exportaciones de manufacturas españolas en sus márgenes extensivo e intensivo. A su vez, cada margen está formado por dos componentes: la entrada de nuevas relaciones comerciales y el valor asociado a las nuevas entradas (para el margen extensivo); y, la supervivencia y el cambio en el valor asociado a las relaciones comerciales que sobreviven (para el margen intensivo). Para ello, aplicamos la metodología propuesta por Besedeš y Prusa (2011).

1 Véase EVENETT y VENABLES (2002), KANG (2004), HUMMELS y KLENOW (2005), FELBERMAYR y KOHLER (2006), AMURGO-PACHECO y PIEROLA (2007), BRENTON y NEWFARMER (2007), HELPMAN et al. (2008) y DE LUCIO et al. (2011).

2 Con este enfoque, MINONDO y REQUENA (2010) analizan el papel de los márgenes de comercio en el crecimiento de las exportaciones de las regiones españolas para el periodo 1988-2006. 
La novedad del trabajo consiste en realizar este análisis por sectores tecnológicos. La motivación para ello descansa en que la composición y la evolución de las exportaciones manufactureras españolas son muy diferentes según el sector tecnológico considerado. Por tanto, consideramos que es importante conocer la contribución de los márgenes de comercio y sus componentes al crecimiento de las exportaciones por sectores tecnológicos. Con este objetivo agrupamos las industrias manufactureras en cuatro sectores que difieren según su contenido tecnológico: bajo, medio-bajo, medio-alto, y alto (siguiendo la clasificación tecnológica de la OCDE).

A partir de los datos de exportaciones bilaterales de productos manufacturados de España y Alemania con el resto del mundo en el periodo 1991-2005, comparamos la evolución de las exportaciones del sector manufacturero español con la de Alemania, con el fin de identificar la fortaleza y debilidad de cada uno de los componentes anteriormente mencionados a la hora de explicar el crecimiento de las exportaciones españolas en relación con Alemania, un país líder en exportación de manufacturas, y especialmente en productos de alto contenido tecnológico. Con este fin realizamos un análisis contra-factual que consiste en determinar cuál habría sido el crecimiento de las exportaciones españolas, en cada sector tecnológico, si la contribución al crecimiento de cada uno de los componentes que conforman los márgenes de comercio hubiera sido la de Alemania (el país de referencia seleccionado).

Los resultados de nuestro trabajo muestran que la importancia de cada componente varía según el sector tecnológico analizado, independientemente del país, así como entre ambos países para un sector tecnológico concreto. Así, los ejercicios contra-factuales indican que el componente de supervivencia es el punto débil del crecimiento de las exportaciones españolas en los cuatro sectores tecnológicos, siendo su importancia creciente con la intensidad tecnológica del sector analizado. Por contra, los resultados apuntan a que los dos componentes del margen extensivo (la tasa de entrada de nuevas relaciones comerciales y su valor de comercio asociado, el valor extensivo) son el punto fuerte de las exportaciones españolas, especialmente en el sector de tecnología media-baja y en el sector de tecnología alta (en los sectores de tecnología baja y media-alta el punto fuerte es la profundización en valor de las relaciones comerciales que sobreviven).

El resto del trabajo se estructura como sigue. En el apartado 2 se describen los datos utilizados. En el apartado 3 se introduce la metodología empleada para descomponer el crecimiento de las exportaciones y se presenta un análisis descriptivo de los márgenes del crecimiento de las exportaciones españolas por sectores tecnológicos (y de sus respectivos componentes). En el apartado 4 se presentan la metodología y los resultados de los ejercicios contra-factuales. Por último, las conclusiones del trabajo quedan recogidas en el apartado 5. 


\section{Datos}

Los datos de exportaciones que se utilizan en este trabajo han sido extraídos de la base de comercio de Naciones Unidas COMTRADE para el periodo 1991-2005, utilizando la clasificación del Sistema Armonizado a 6 dígitos. España y Alemania pueden exportar hasta 5014 productos a un total de 188 países durante el periodo de análisis. Los valores de exportación han sido deflactados utilizando el deflactor de las exportaciones por sectores tecnológicos de la OCDE (año base 2005).

Los productos del Sistema Armonizado han sido clasificados en cuatro sectores tecnológicos (sectores de bajo, medio-bajo, medio-alto y alto contenido tecnológico) siguiendo la clasificación de la OCDE CUCI (rev. 3 a 4-dígitos). La correspondencia entre productos del Sistema Armonizado y los sectores CUCI se ha obtenido de EUROSTAT-RAMON. En el Cuadro del Apéndice se muestra la clasificación de los distintos sectores manufactureros en los cuatro sectores tecnológicos, según la clasificación CUCI propuesta por la OCDE.

En los Cuadros 1, 2 y 3 se presenta un análisis descriptivo de la evolución de las exportaciones para España y para Alemania, para el periodo 1991-2005. Si observamos la evolución temporal de la participación de cada sector en las exportaciones totales de manufacturas de España (Cuadro 1), podemos concluir que el sector más importante, por volumen de comercio, es el de tecnología media-alta, con un peso promedio del 47,16 por 100 durante el periodo, mientras que el sector con menor peso es el de contenido tecnológico alto, con un peso promedio del 10,13 por 100 . Para Alemania (Cuadro 2), el sector más importante también es el de tecnología media-alta (51,38 por 100 en media), aunque el segundo en importancia es el de tecnología alta (17,92 por 100), siendo el sector menos importante el de tecnología baja $(15,24$ por 100$)$.

La participación de cada sector tecnológico en las exportaciones totales se comporta de forma bastante estable para el caso español. Sin embargo, en el caso alemán (Cuadro 2) esta estabilidad se observa en los sectores de tecnología media-baja y media-alta, pero no en los sectores de tecnología baja o alta. En particular, se observa una disminución en la participación de las exportaciones de sectores de baja tecnología que es absorbida por un aumento de la participación en las exportaciones de los sectores de tecnología alta. Por otra parte, comparando los Cuadros 1 y 2 también podemos ver las diferencias en la composición de las exportaciones de manufacturas entre España y Alemania. En particular, España está relativamente más concentrada que Alemania en exportaciones de manufacturas de bajo contenido tecnológico y sectores de tecnología media-baja ( 23,39 por 100 y 19,32 por 100 , respectivamente, para España, frente a 15,24 por 100 y 15,45 por 100 para Alemania). Por el contrario, en Alemania se observa una mayor concentración que en España en la exportación de productos de sectores de tecnología media-alta y alta $(51,38$ por 100 y 17,92 por 100 , respectivamente, para Alemania, frente a 47,16 por 100 y 10,13 por 100 para España). De hecho, las diferencias más grandes en la composición de las exportaciones de manufacturas entre España y Alemania se encuentran en los 
CUADRO 1

DISTRIBUCIÓN DE LAS EXPORTACIONES ESPAÑOLAS DE MANUFACTURAS POR SECTORES TECNOLÓGICOS (1991-2005)

\begin{tabular}{|c|c|c|c|c|}
\hline Años & $\begin{array}{c}\text { Tecnología } \\
\text { baja (\%) }\end{array}$ & $\begin{array}{c}\text { Tecnología } \\
\text { media-baja (\%) }\end{array}$ & $\begin{array}{c}\text { Tecnología } \\
\text { media-alta (\%) }\end{array}$ & $\begin{array}{c}\text { Tecnología } \\
\text { alta (\%) }\end{array}$ \\
\hline $\mathbf{1 9 9 1}$ & 22,70 & 22,45 & 45,34 & 9,51 \\
$\mathbf{1 9 9 2}$ & 22,08 & 21,47 & 47,23 & 9,22 \\
$\mathbf{1 9 9 3}$ & 22,41 & 22,50 & 45,14 & 9,96 \\
$\mathbf{1 9 9 4}$ & 22,92 & 20,61 & 46,43 & 10,04 \\
$\mathbf{1 9 9 5}$ & 23,26 & 20,21 & 47,58 & 8,95 \\
$\mathbf{1 9 9 6}$ & 23,73 & 18,72 & 48,14 & 9,41 \\
$\mathbf{1 9 9 7}$ & 24,75 & 18,93 & 47,26 & 9,06 \\
$\mathbf{1 9 9 8}$ & 24,25 & 17,76 & 48,51 & 9,48 \\
$\mathbf{1 9 9 9}$ & 23,76 & 17,49 & 48,30 & 10,45 \\
$\mathbf{2 0 0 0}$ & 23,35 & 17,36 & 48,70 & 10,59 \\
$\mathbf{2 0 0 1}$ & 24,64 & 16,84 & 47,96 & 10,56 \\
$\mathbf{2 0 0 2}$ & 24,56 & 17,71 & 46,56 & 11,17 \\
$\mathbf{2 0 0 3}$ & 23,58 & 17,78 & 47,38 & 11,27 \\
$\mathbf{2 0 0 4}$ & 22,83 & 19,31 & 47,08 & 10,78 \\
$\mathbf{2 0 0 5}$ & 22,06 & 20,64 & 45,76 & 11,54 \\
\hline Media & $\mathbf{2 3 , 3 9}$ & $\mathbf{1 9 , 3 2}$ & 47,16 & $\mathbf{1 0 , 1 3}$ \\
\hline
\end{tabular}

\section{CUADRO 2}

DISTRIBUCIÓN DE LAS EXPORTACIONES ALEMANAS DE MANUFACTURAS POR SECTORES TECNOLÓGICOS (1991-2005)

\begin{tabular}{|l|c|c|c|c|}
\hline $\mathbf{A n ̃ o s}$ & $\begin{array}{c}\text { Tecnología } \\
\text { baja (\%) }\end{array}$ & $\begin{array}{c}\text { Tecnología } \\
\text { media-baja (\%) }\end{array}$ & $\begin{array}{c}\text { Tecnología } \\
\text { media-alta (\%) }\end{array}$ & $\begin{array}{c}\text { Tecnología } \\
\text { alta (\%) }\end{array}$ \\
\hline $\mathbf{1 9 9 1}$ & 17,27 & 17,12 & 50,12 & 15,49 \\
$\mathbf{1 9 9 2}$ & 17,46 & 16,66 & 50,80 & 15,08 \\
$\mathbf{1 9 9 3}$ & 17,21 & 16,46 & 50,84 & 15,48 \\
$\mathbf{1 9 9 4}$ & 16,62 & 16,04 & 51,69 & 15,65 \\
$\mathbf{1 9 9 5}$ & 16,10 & 16,43 & 52,04 & 15,43 \\
$\mathbf{1 9 9 6}$ & 16,11 & 15,06 & 53,28 & 15,55 \\
$\mathbf{1 9 9 7}$ & 15,43 & 15,21 & 52,55 & 17,62 \\
$\mathbf{1 9 9 8}$ & 15,15 & 15,02 & 52,21 & 19,63 \\
$\mathbf{1 9 9 9}$ & 14,78 & 14,03 & 51,56 & 20,97 \\
$\mathbf{2 0 0 0}$ & 13,77 & 14,70 & 50,56 & 20,40 \\
$\mathbf{2 0 0 1}$ & 13,85 & 14,57 & 50,18 & 19,55 \\
$\mathbf{2 0 0 2}$ & 14,12 & 14,74 & 50,89 & 20,19 \\
$\mathbf{2 0 0 3}$ & 13,98 & 14,61 & 51,86 & 19,69 \\
$\mathbf{2 0 0 4}$ & 13,38 & 15,20 & 51,23 & $\mathbf{1 7 , 9 2}$ \\
$\mathbf{2 0 0 5}$ & 13,42 & 15,96 & 50,93 & \\
\hline Media & $\mathbf{1 5 , 2 4}$ & $\mathbf{1 5 , 4 5}$ & $\mathbf{5 1 , 3 8}$ & \\
\hline
\end{tabular}


extremos tecnológicos. Mientras que el porcentaje de exportaciones españolas en sectores de tecnología baja está por encima del alemán en 8,15 puntos porcentuales, el porcentaje de exportaciones españolas en sectores de tecnología alta está por debajo del alemán en 7,79 puntos porcentuales. En tecnología media (incluyendo media-baja y media-alta) los dos países son muy similares, aunque España está relativamente más orientada hacia los sectores de tecnología media-baja.

Para completar la comparación entre la evolución de las exportaciones manufactureras de España y Alemania, en el Cuadro 3 podemos ver que las exportaciones españolas representan un 21,94 por 100, en promedio, de las exportaciones alemanas. Analizando esta información por sectores tecnológicos, vemos que el porcentaje para el sector de tecnología media-alta está cercano a la media (las exportaciones españolas para este sector representan un 20,15 por 100 de las alemanas), el porcentaje es superior a la media en los sectores de tecnología baja y de tecnología mediabaja $(34,19$ por 100 y 27,33 por 100 , respectivamente) y está por debajo de la media en el sector de tecnología alta (12,46 por 100 de las exportaciones alemanas para el mismo grupo tecnológico).

CUADRO 3

TAMAÑO RELATIVO DE LAS EXPORTACIONES ESPAÑOLAS RESPECTO A LAS EXPORTACIONES DE ALEMANIA (TOTAL Y POR SECTORES TECNOLÓGICOS)

\begin{tabular}{|c|c|c|c|c|c|}
\hline Años & $\begin{array}{c}\text { Total } \\
\mathbf{( \% )}\end{array}$ & $\begin{array}{c}\text { Tecnología } \\
\text { baja (\%) }\end{array}$ & $\begin{array}{c}\text { Tecnología } \\
\text { media-baja (\%) }\end{array}$ & $\begin{array}{c}\text { Tecnología } \\
\text { media-alta (\%) }\end{array}$ & $\begin{array}{c}\text { Tecnología } \\
\text { alta (\%) }\end{array}$ \\
\hline $\mathbf{1 9 9 1}$ & 19,44 & 25,54 & 25,49 & 17,59 & 11,93 \\
$\mathbf{1 9 9 2}$ & 18,94 & 23,95 & 24,40 & 17,61 & 11,58 \\
$\mathbf{1 9 9 3}$ & 20,30 & 26,43 & 27,76 & 18,03 & 13,06 \\
$\mathbf{1 9 9 4}$ & 21,25 & 29,30 & 27,30 & 19,09 & 13,64 \\
$\mathbf{1 9 9 5}$ & 21,02 & 30,38 & 25,87 & 19,22 & 12,19 \\
$\mathbf{1 9 9 6}$ & 22,79 & 33,57 & 28,34 & 20,60 & 13,79 \\
$\mathbf{1 9 9 7}$ & 23,87 & 38,28 & 29,71 & 21,47 & 12,87 \\
$\mathbf{1 9 9 8}$ & 22,79 & 36,48 & 26,95 & 21,18 & 12,26 \\
$\mathbf{1 9 9 9}$ & 24,00 & 38,57 & 29,93 & 22,49 & 12,78 \\
$\mathbf{2 0 0 0}$ & 23,79 & 40,33 & 28,10 & 22,91 & 12,02 \\
$\mathbf{2 0 0 1}$ & 22,38 & 39,81 & 25,86 & 21,39 & 11,05 \\
$\mathbf{2 0 0 2}$ & 22,29 & 38,76 & 26,78 & 20,40 & 12,29 \\
$\mathbf{2 0 0 3}$ & 23,37 & 39,41 & 28,43 & 21,35 & 13,47 \\
$\mathbf{2 0 0 4}$ & 22,18 & 37,84 & 28,16 & 20,38 & 11,84 \\
$\mathbf{2 0 0 5}$ & 20,75 & 34,11 & 26,83 & 18,64 & 12,16 \\
\hline Media & $\mathbf{2 1 , 9 4}$ & $\mathbf{3 4 , 1 9}$ & $\mathbf{2 7 , 3 3}$ & $\mathbf{2 0 , 1 5}$ & $\mathbf{1 2 , 4 6}$ \\
\hline
\end{tabular}


Finalmente, en el Cuadro 4 presentamos la tasa de crecimiento medio anual de las exportaciones de manufacturas de España y Alemania para el periodo considerado. A lo largo del periodo las exportaciones españolas han crecido más rápidamente que las alemanas en los cuatro sectores tecnológicos, aunque con diferencias importantes: en el sector de contenido tecnológico alto la diferencia en el crecimiento medio anual es pequeña $(6,17$ por 100 versus 6,03 por 100) comparada con los otros tres sectores, donde la diferencia supera los dos puntos para el sector de tecnología baja (4,58 por 100 versus 2,52 por 100$)$, y está alrededor del medio punto cada año en el caso de los sectores de tecnología media-alta y media-baja.

\section{CUADRO 4}

TASA DE CRECIMIENTO ANUAL DE LAS EXPORTACIONES (REALES) DE MANUFACTURAS POR SECTORES TECNOLÓGICOS (1991-2005)

\begin{tabular}{|c|c|c|c|c|}
\hline & $\begin{array}{c}\text { Tecnología } \\
\text { baja (\%) }\end{array}$ & $\begin{array}{c}\text { Tecnología } \\
\text { media-baja (\%) }\end{array}$ & $\begin{array}{c}\text { Tecnología } \\
\text { media-alta (\%) }\end{array}$ & $\begin{array}{c}\text { Tecnología } \\
\text { alta (\%) }\end{array}$ \\
\hline España & 4,58 & 4,19 & 4,85 & 6,17 \\
Alemania & 2,52 & 3,82 & 4,44 & 6,03 \\
\hline
\end{tabular}

Así pues, en las exportaciones manufactureras españolas tienen un peso mayor los productos de bajo contenido tecnológico que en las exportaciones alemanas y el diferencial de crecimiento por sectores confirma la mayor facilidad que tiene España para exportar este tipo de productos frente a Alemania. Sin embargo, es un dato positivo que las exportaciones españolas de productos de contenido tecnológico más alto hayan crecido más que las exportaciones de productos de contenido tecnológico más bajo, lo que ha permitido una lenta redistribución del peso de las exportaciones españolas hacia los sectores de contenido tecnológico medio-alto y alto.

\section{Los márgenes del comercio}

\subsection{Metodología}

Antes de proceder a la descomposición del crecimiento de las exportaciones españolas en el margen extensivo e intensivo, es importante explicar cierta terminología utilizada en el trabajo. En primer lugar, definimos una relación comercial como cada una de las transacciones que realiza un país con otro país de un producto concreto durante un periodo de tiempo medido en años. Toda relación comercial nace en algún momento, sobrevive durante un periodo de tiempo y, quizás, acaba interrumpiéndose. Por lo tanto, una relación comercial tiene cuatro dimensiones que la caracterizan (origen del exportador, origen del importador, producto y número de años que existe). En otras palabras, para un país exportador determinado, el par país 
importador-producto durante el lapso de tiempo que persiste la relación define una relación comercial. Este modo de definir una relación comercial facilita el cálculo de las tasas de supervivencia y de profundización de las relaciones comerciales, al existir una relación unívoca entre una relación comercial y el lapso de tiempo que tiene dicha relación.

El margen extensivo se define como el número de nuevas relaciones comerciales que tiene un país (entrada) y su valor (valor extensivo) ${ }^{3}$. Esta definición permite todas las formas en las que el margen extensivo puede variar. Por ejemplo, el margen extensivo de un país exportador aumenta cuando se vende un determinado producto a un nuevo país, cuando se vende un nuevo producto a un país al que ya se exportaba o cuando se vende un nuevo producto a un nuevo país. Definimos también el valor del margen extensivo como el valor de las exportaciones asociadas a un cambio en el margen extensivo provocado por la aparición de nuevas relaciones comerciales.

Análogamente, en este trabajo describimos el margen intensivo a través de sus dos dimensiones, la supervivencia (cuánto tiempo dura una relación comercial) y la profundización (cambio en el valor de las exportaciones de las relaciones comerciales que sobreviven). Para ello, llamamos «tiempo de supervivencia» al periodo que mide cuánto tiempo se mantiene activa una relación comercial. En un año cualquiera, hay una mezcla de relaciones comerciales con diferentes duraciones (medidas en años), y la distribución de dichas duraciones refleja la longevidad de las relaciones existentes ese año.

Con el fin de examinar el papel de los márgenes extensivo e intensivo en el crecimiento de las exportaciones utilizamos la metodología propuesta por Besedeš y Prusa (2011), que permite descomponer el crecimiento de las exportaciones en los distintos componentes que forman el margen extensivo (nuevas relaciones y su valor extensivo) y el margen intensivo (supervivencia y profundización). Una novedad en nuestro trabajo consiste en permitir que cada uno de los componentes tenga un impacto diferente en cada sector tecnológico.

Así, tenemos que para cada año $t$ podemos representar el valor total de las exportaciones de un sector tecnológico $z, Z=\{$ bajo, medio-bajo, medio-alto, alto $\}$, como:

$$
V_{z, t}=n_{z, t} v_{z, t}
$$

donde $V_{z, t}$ es el valor de las exportaciones totales en el año $t$ para el sector tecnológico $z, n_{z, t}$ es el número de relaciones comerciales en $t$, y $v_{z, t}$ es el valor medio de las exportaciones de cada relación comercial en el año $t$. A las relaciones comerciales en $t$ que han sobrevivido entre $t-1$ y $t$ las llamamos $s_{z, t}$, y a las relaciones comerciales de nueva creación las llamamos $e_{z, t}$, de modo que $n_{z, t}=s_{z, t}+e_{z, t}$.

3 Véase HUMMELS y KLENOW (2005) y EATON et al . (2007) para definiciones similares del margen extensivo. 
Utilizando esta notación, el cambio en el valor total de las exportaciones del sector $z$, en el periodo de $t$ a $t+1$, se puede escribir como:

$$
\begin{aligned}
G_{z, t+1} & =V_{z, t+1}-V_{z, t}=n_{z, t+1} v_{z, t+1}-n_{z, t} v_{z, t}= \\
& =\left(s_{z, t+1}+e_{z, t+1}\right) v_{z, t+1}-\left(s_{z, t}+e_{z, t}\right) v_{z, t}= \\
& =s_{z, t+1} v_{z, t+1}+e_{z, t+1} v_{z, t+1}-s_{z, t+1} v_{z, t}-d_{z, t} v_{z, t}= \\
& =s_{z, t+1}\left(v_{z, t+1}-v_{z, t}\right)-d_{z, t} v_{z, t}+e_{z, t+1} v_{z, t+1}
\end{aligned}
$$

donde $s_{z, t+1}$ es el número de relaciones comerciales en el sector tecnológico $z$ que sobreviven entre $t$ y $t+1,\left(v_{z, t+1}-v_{z, t}\right)$ es el crecimiento de las exportaciones para cada relación comercial que sobrevive, $d_{z, t}$ es el número de relaciones comerciales que desaparecen en $t$, siendo $d_{z, t} v_{z, t}$ su valor total, y $e_{z, t+1}$ es el número de nuevas relaciones comerciales, siendo $e_{z, t+1} v_{z, t+1}$ su valor total.

Siguiendo a Besedeš y Prusa (2011), podemos refinar la descomposición del crecimiento de las exportaciones en cada sector tecnológico $z$, considerando que la probabilidad de que una relación comercial concreta termine en el año $t$ depende de la edad de dicha relación comercial (en particular, se espera que la probabilidad de que una relación comercial acabe esté negativamente correlacionada con el tiempo que ha sobrevivido hasta ese momento, es decir, esperamos que exista lo que en literatura econométrica se conoce como «negative duration dependence»). Para ello, necesitamos incorporar, en la descomposición realizada, la edad que tiene cada relación comercial. Además, podemos permitir que las funciones de riesgo y supervivencia de las relaciones comerciales sean específicas a cada industria $j$ dentro de un sector tecnológico $z$ (véase Cuadro del Apéndice). Tomando en consideración estos dos refinamientos, podemos definir:

$$
\begin{aligned}
s_{z j, t} & \equiv\left\{s_{z j, t}^{0}, s_{z j, t}^{1}, s_{z j, t}^{2}, \ldots, s_{z j, t}^{i}, \ldots, s_{z j, t}^{I}\right\} \\
d_{z j, t} & \equiv\left\{d_{z j, t}^{0}, d_{z j, t}^{1}, d_{z j, t}^{2}, \ldots, d_{z j, t}^{i}, \ldots, d_{z j, t}^{I}\right\} \\
v_{z j, t} & \equiv\left\{v_{z j, t}^{0}, v_{z j, t}^{1}, v_{z j, t}^{2}, \ldots, v_{z j, t}^{i}, \ldots, v_{z j, t}^{I}\right\} \\
h_{z j, t} & \equiv\left\{h_{z j, t}^{0}, h_{z j, t}^{1}, h_{z j, t}^{2}, \ldots, h_{z j, t}^{i}, \ldots, h_{z j, t}^{I}\right\}
\end{aligned}
$$

donde el superíndice $i$ denota la edad en la que se encuentra una relación comercial (años de supervivencia), y $h_{z j, t}$ denota la tasa de riesgo de terminación de una relación comercial en la industria $j$ que pertenece al sector tecnológico $z$ y que ha sobrevivido hasta $t$.

Ahora, podemos re-escribir la ecuación (1) de la siguiente manera:

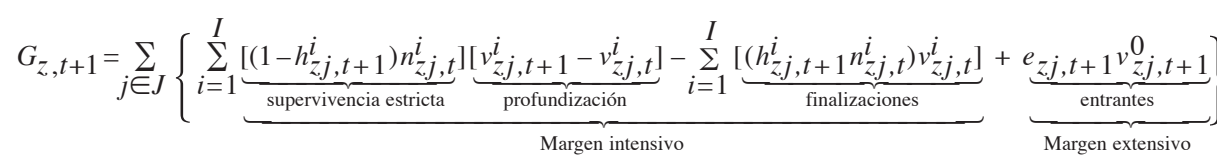


donde $I$ denota la edad máxima potencial de una relación comercial; $\left(1-h_{z j, t+1}^{i}\right)$ nos da el porcentaje de relaciones comerciales que sobreviven entre $t$ y $t+1$ en el sector tecnológico $z ;\left(1-h_{z j, t+1}^{i}\right) n_{z j, t}^{i}$ representa el número total de relaciones comerciales que sobreviven entre $t$ y $t+1$ en el sector tecnológico $z$ y que corresponden a relaciones comerciales que continúan ininterrumpidamente durante $i$ años (elemento de supervivencia estricta que forma parte del componente general de supervivencia del margen intensivo); $\left[v_{z j, t+1}^{i}-v_{z j, t}^{i}\right]$ representa la profundización o el crecimiento del valor de comercio que se produce en el sector tecnológico $z$ para relaciones comerciales que sobreviven entre $t$ y $t+1 ;\left(h_{z j, t+1}^{i} n_{z j, t}^{i}\right)$ proporciona el número de relaciones comerciales que finalizan entre $t \mathrm{y} t+1 ;\left(h_{z j, t+1}^{i} n_{z j, t}^{i}\right) v_{z j, t}^{i}$ indica su valor total de comercio (elemento de finalizaciones que junto con el de supervivencia estricta conforma el componente general de supervivencia del margen intensivo); y $e_{z j, t+1} v_{z j, t+1}^{0}$ indica el valor de comercio que corresponde a las relaciones comerciales en $t+1$ de nueva creación (entradas).

\subsection{El margen extensivo}

En el Cuadro 5 caracterizamos de un modo descriptivo el papel del margen extensivo en el crecimiento de las exportaciones por sectores tecnológicos para España y Alemania durante el periodo 1991-2005. La primera columna muestra el crecimiento de las exportaciones totales mientras que la segunda columna muestra el crecimiento del número de relaciones comerciales. En el caso de España, se aprecia una relación más estrecha entre ambas tasas de crecimiento que en el caso de Alemania. También se observa que la diferencia entre el crecimiento de las exportaciones y el crecimiento del número de relaciones comerciales es mayor en el caso de Alemania que en el caso de España, siendo esto más acusado en el sector de productos de tecnología media-alta y alta. Los resultados en las columnas 1 y 2 para Alemania indican que el margen intensivo puede desempeñar un papel más importante que el margen extensivo ya que las exportaciones crecen mucho más rápido que las relaciones de exportación, un resultado general anticipado por Besedeš y Prusa (2011) para países desarrollados. Para el caso de España no encontramos cambios significativos entre sectores, a excepción del sector de tecnología alta, para el que el crecimiento de las exportaciones es mayor. Para Alemania se observan mayores diferencias entre sectores $y$, además, se observa que el crecimiento de las exportaciones aumenta conforme aumenta la intensidad tecnológica del sector, mientras que el número de relaciones comerciales está inversamente relacionado con la intensidad tecnológica del sector. El sector de tecnología alta es, además, el de mayor tasa de crecimiento de las exportaciones.

La columna 3 informa sobre uno de los componentes del margen extensivo: la tasa de entrada. Para cada sector tecnológico (en cada país y en cada año $t$ ), calculamos la tasa de entrada como $e_{z, t} / n_{z, t}$ donde $e_{z, t}$ es el número de relaciones comerciales que se inician en $t$ y $n_{z, t}$ es el número de relaciones comerciales totales en $t$. 
Como puede observarse, este componente del margen extensivo es bastante elevado, especialmente en el caso de España, con un porcentaje de nuevas relaciones cada año entre el 27,4 por 100 y el 29,3 por 100 (en el caso de Alemania este porcentaje varía entre el 16,0 por 100 y el 19,3 por 100). Estos valores apuntan a que el margen extensivo puede estar desempeñando un papel más importante en la dinámica de crecimiento de las exportaciones españolas que en la de las alemanas, ya que en promedio las tasas de entrada son 1,5 veces mayores en España.

La última columna del Cuadro 5 presenta el segundo componente del margen extensivo, es decir, el valor del margen extensivo. Éste se ha calculado como $\left(v_{z, t}^{0} e_{z, t}\right) /\left(v_{z, t} n_{z, t}\right)$, donde $v_{z, t}^{0}$ es el valor promedio de las exportaciones de las relaciones comerciales que se inician en el año $t$ y $v_{z, t}$ es el valor promedio de las exportaciones de las relaciones comerciales en el año $t$. El patrón que observamos es similar al de la columna anterior: comparado con Alemania, el valor del margen extensivo es mayor para España en los cuatro sectores tecnológicos. Sin embargo, el valor de comercio de las nuevas relaciones comerciales en ambos países es mucho menor que el de las relaciones comerciales ya establecidas. Esto apunta al hecho de que las nuevas relaciones comerciales sólo pueden tener un impacto importante sobre el crecimiento de las exportaciones si sobreviven en el tiempo y su valor de comercio se consolida, ya que en sus primeros años muestran unos valores muy pequeños como para que puedan tener un impacto significativo sobre el crecimiento agregado de las exportaciones, independientemente del sector analizado.

\section{CUADRO 5}

MARGEN EXTENSIVO DE LAS EXPORTACIONES DE MANUFACTURAS POR SECTORES TECNOLÓGICOS, ESPAÑA Y ALEMANIA, 1991-2005

\begin{tabular}{|l|c|c|c|c|}
\hline & $\begin{array}{c}\text { Crecimiento de } \\
\text { las exportaciones } \\
\text { (términos reales) }\end{array}$ & $\begin{array}{c}\text { Crecimiento del } \\
\text { número de } \\
\text { relaciones de } \\
\text { comercio } \\
\text { (producto-país) }\end{array}$ & $\begin{array}{c}\text { Margen } \\
\text { extensivo } \\
\text { (entrada) }\end{array}$ & $\begin{array}{c}\text { Margen } \\
\text { extensivo } \\
\text { (valor extensivo) }\end{array}$ \\
\hline España & & & & \\
Bajo & 4,58 & 5,90 & 27,82 & 2,14 \\
Medio-bajo & 4,19 & 4,19 & 27,39 & 6,38 \\
Medio-alto & 4,85 & 4,74 & 27,74 & 2,51 \\
Alto & 6,17 & 5,02 & 29,28 & 5,43 \\
Alemania & 2,52 & & & \\
Bajo & 3,82 & 2,44 & 19,29 & 1,50 \\
Medio-bajo & 4,44 & 1,60 & 17,39 & 2,78 \\
Medio-alto & 6,03 & 1,44 & 16,02 & 1,11 \\
Alto & & 16,46 & 1,45 \\
\hline
\end{tabular}




\subsection{El margen intensivo}

Por lo que respecta al análisis descriptivo del componente de supervivencia del margen intensivo (que como ya se vio en la ecuación [2] se compone de dos elementos, supervivencia estricta y finalizaciones), a través de técnicas de análisis de duración podemos obtener una representación apropiada y precisa de la duración media de las relaciones comerciales para un determinado año. Así, en este trabajo estimamos funciones de supervivencia Kaplan-Meier para los cuatro sectores tecnológicos tanto para España como para Alemania (véase Gráfico 1).

La principal conclusión que se desprende del Gráfico 1 es que las funciones de supervivencia de España y Alemania son diferentes para los cuatro sectores tecnológicos analizados ${ }^{4}$. La duración de las relaciones comerciales para los cuatro sectores tecnológicos analizados es más corta para España que para Alemania. La duración mediana es de un año en España y de dos años en Alemania. Esto significa que el 50 por 100 de las relaciones comerciales en España desaparecen al final de su primer año. En Alemania esto ocurre el segundo año.

A continuación, podemos ver en el Gráfico 1 que a medida que aumenta la intensidad tecnológica, la brecha de supervivencia entre las duraciones de las relaciones comerciales de España y Alemania se va ampliando. En concreto, el 25 por 100 de las relaciones comerciales en el sector de baja tecnología sobrevive seis años en Alemania pero sólo cuatro años en España; en el sector de tecnología media-baja, siete años en Alemania frente a cuatro en España; en el sector de tecnología media-alta, ocho años en Alemania frente a tres en España; y, finalmente, en el sector de tecnología alta, siete años en Alemania frente a tres en España. Por lo tanto, incluso en el sector donde España revela una ventaja comparativa superior a la de Alemania, como es el sector de tecnología baja, las relaciones comerciales son más cortas que en Alemania, aspecto que desempeñará un papel importante en la descomposición del crecimiento de las exportaciones.

En resumen, aunque encontramos que la mayoría de relaciones comerciales tiende a durar poco para los dos países considerados, existen algunas diferencias importantes entre sectores tecnológicos entre países. En Alemania, las relaciones comerciales sobreviven más tiempo a medida que aumenta la intensidad tecnológica del sector. Sin embargo, en el caso de España no es así, ya que las tasas de supervivencia son bastante similares entre sectores y, en todo caso, el sector de alta tecnología es el que presenta las menores tasas de supervivencia.

El análisis de la duración de las relaciones comerciales nos ayuda a entender el efecto del margen extensivo y del margen intensivo sobre el crecimiento de las exportaciones. Si existe una elevada tasa de fracaso entre las relaciones comerciales

\footnotetext{
4 A través del contraste log-rank podemos rechazar la hipótesis nula de la misma función de supervivencia para España y Alemania en los sectores de baja, media-baja, media-alta y alta tecnología, con unos valores de $\chi^{2}=4.256,06$ y un $p$-value $=0,000 ; \chi^{2}=12,823,43$ y un $p$-value $=0,000 ; \chi^{2}=5.179,84$ y un $p$-value $=0,000 ; \mathrm{y} \chi^{2}=5.253,31$ y un $p$-value $=0,000$, respectivamente.
} 


\section{GRÁFICO 1}

\section{ANÁLIS IS NO PARAMÉTRICO DE LA SUPERVIVENCIA DE LAS RELACIONES COMERCIALES, ESPAÑA Y ALEMANIA}
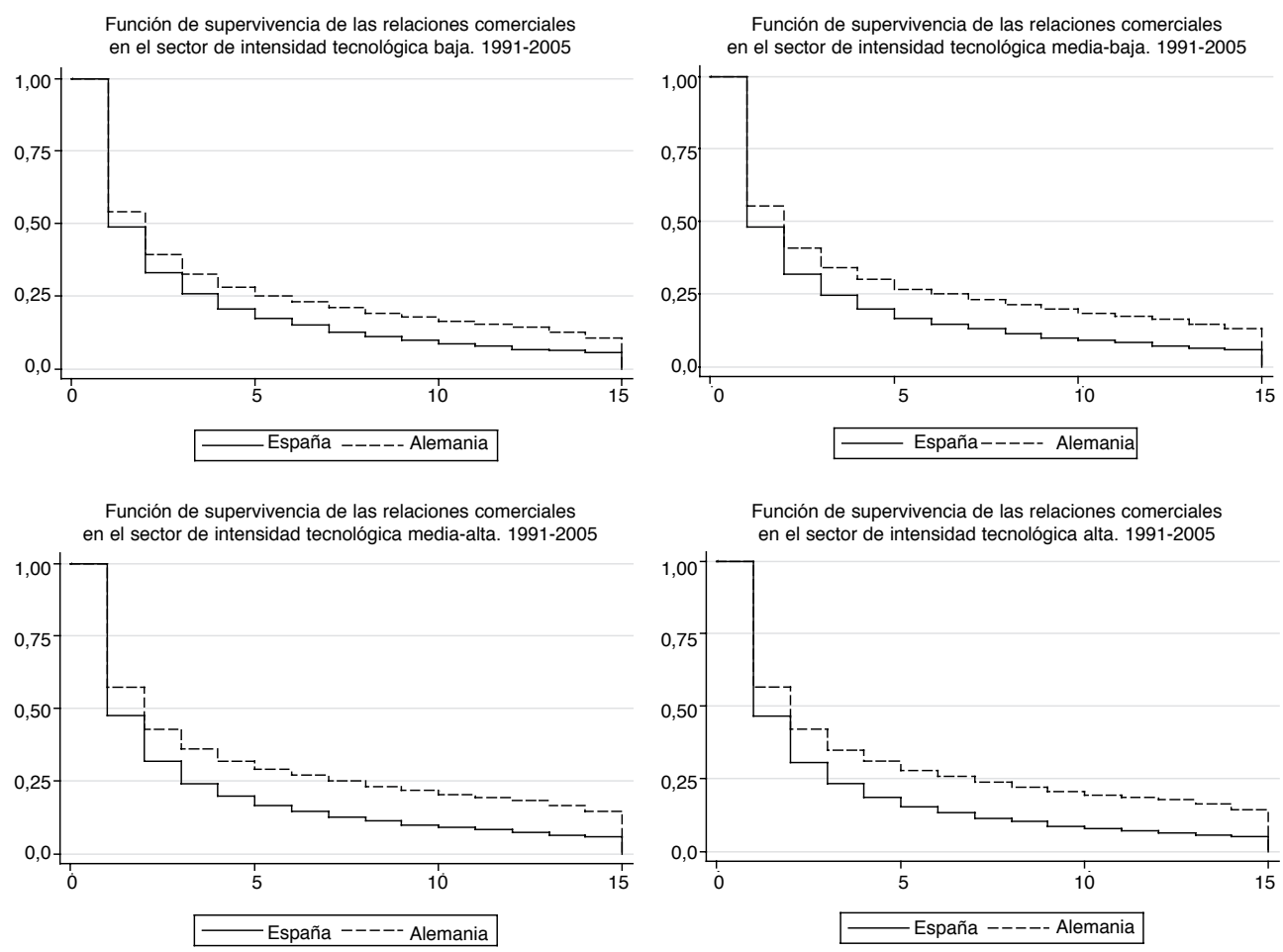

nuevas, eso implica que el aumento del margen extensivo tendrá al cabo de unos pocos años una escasa contribución al crecimiento de las exportaciones. Al mismo tiempo, si las relaciones comerciales desaparecen muy pronto, esto afectará negativamente a la contribución del margen intensivo al crecimiento de las exportaciones, ya que la profundización de las relaciones comerciales está condicionada a la propia supervivencia de las mismas.

El análisis descriptivo del componente de profundización del margen intensivo aparece en el Cuadro 6. En las columnas 1 a 3 analizamos la profundización de las relaciones de largo plazo, esto es, relaciones comerciales que han sobrevivido desde 1991 hasta 2005. La columna 1 contiene la fracción de las relaciones de 2005 que estaban ya activas en 1991 (en media, el 39,4 por 100 de las relaciones comerciales alemanas son de largo plazo, frente al 18,5 por 100 de las españolas, algo menos de la mitad que las alemanas). La columna 2 presenta el porcentaje en las exportaciones de 2005 que representan las relaciones de largo plazo de la columna 1: la mayoría del valor de comercio pertenece a relaciones de largo plazo (74,50 por 100 de media en España y 81,5 por 100 de media en Alemania). La menor profundización en el valor de las exportaciones de 
las relaciones comerciales de largo plazo de España, en relación a Alemania, vuelve a señalar al componente de supervivencia como el elemento clave del crecimiento diferencial de las exportaciones entre España y Alemania. En la columna 3, presentamos la tasa media anual de crecimiento de la profundización de las relaciones de largo plazo. Es importante resaltar el hecho de que las relaciones comerciales españolas de largo plazo crecen en valor más rápidamente que las alemanas, especialmente en los sectores de contenido tecnológico bajo y medio-alto.

\section{CUADRO 6}

«PROFUNDIZACIÓN» EXPORTADORA DE LAS RELACIONES COMERCIALES QUE SOBREVIVEN POR SECTOR TECNOLÓGICO ENTRE 1991 Y 2005

\begin{tabular}{|l|c|c|c|}
\hline \multirow{2}{*}{$\begin{array}{c}\text { País } \\
\text { Sector tecnológico }\end{array}$} & $\begin{array}{c}\text { Fracción de las } \\
\text { relaciones de 2005 } \\
\text { activas en 1991 (\%) }\end{array}$ & $\begin{array}{c}\text { Su fracción en el } \\
\text { valor de comercio } \\
\text { de 2005 (\%) }\end{array}$ & $\begin{array}{c}\text { Crecimiento } \\
\text { del valor } \\
\text { de comercio (\%) }\end{array}$ \\
\cline { 2 - 4 } & & & \\
España & 17,01 & 78,01 & 5,14 \\
Bajo & 20,12 & 62,04 & 4,24 \\
Medio-bajo & 19,74 & 79,44 & 5,51 \\
Medio-alto & 17,09 & 78,25 & 4,73 \\
Alto & 33,94 & 81,87 & 1,56 \\
Alemania & 40,31 & 74,18 & 3,83 \\
Bajo & 42,27 & 86,33 & 3,55 \\
Medio-bajo & 42,28 & 83,49 & 4,17 \\
Medio-alto & & & \\
Alto & & & \\
\hline
\end{tabular}

El Gráfico 2 ofrece una visión diferente del componente de profundización que no tiene la limitación de considerar solamente las relaciones comerciales que sobreviven el periodo completo (tal y como hacíamos en el Cuadro 6). Este enfoque ofrece una perspectiva general de la profundización para el periodo en su conjunto. Esto es así porque el cálculo del valor de comercio se realiza condicionando a relaciones comerciales que sobreviven al menos $k$ años y calculando a continuación, para cada año que sobreviven, el porcentaje del valor de las exportaciones españolas sobre las alemanas (para cada sector tecnológico). En el Gráfico 2 todos los ratios son inferiores a 1, lo que revela que la profundización de las exportaciones de manufacturas españolas, con independencia del sector y del año en que se encuentren, es siempre inferior a la alemana. Además, en general, cuanto mayor es el contenido tecnológico del sector, menor es la tasa de profundización relativa de las exportaciones españolas. Esto indica que la profundización también jugará un papel importante a la hora de explicar el crecimiento diferencial de las exportaciones españolas respecto a las alemanas, especialmente en sectores de alto contenido tecnológico. 


\section{GRÁFICO 2}

TAMAÑO MEDIO RELATIVO DE LAS RELACIONES DE EXPORTACIÓN ESPAÑOLAS QUE SOBREVIVEN K AÑOS, EN RELACIÓN A LAS ALEMANAS, POR SECTORES TECNOLÓGICOS

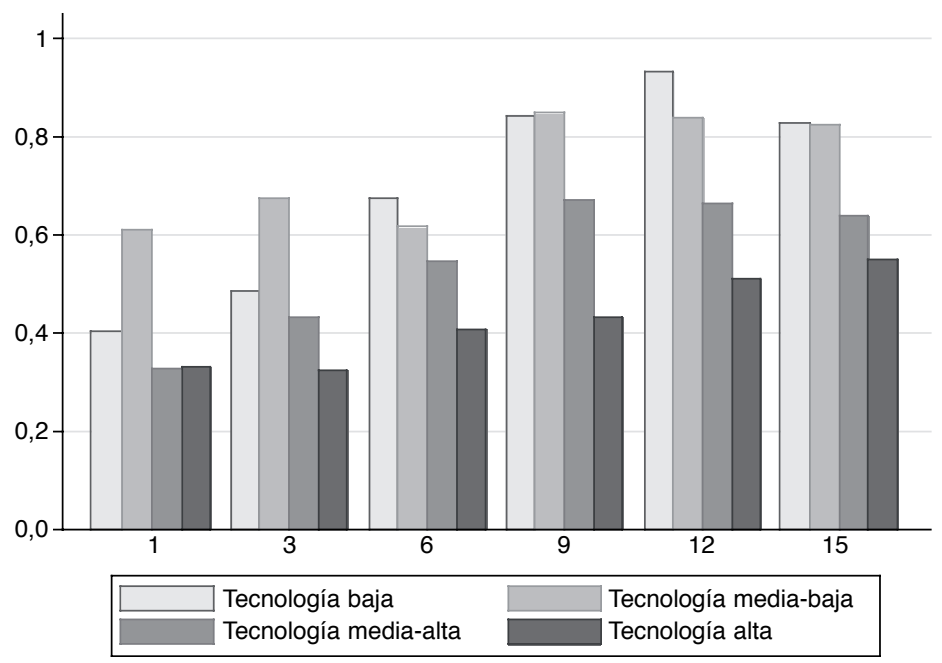

\section{Descomposición del crecimiento de las exportaciones: ejercicios contra-factuales}

\subsection{Metodología}

En esta sección presentamos un ejercicio contra-factual con el fin de evaluar qué componentes explican mejor el diferencial de crecimiento de las exportaciones españolas respecto a las alemanas en cada uno de los sectores tecnológicos. Para llevar a cabo estos ejercicios contra-factuales, a partir de la ecuación [2] sustituiremos cada uno de los cuatro componentes descritos en la sección anterior (entrada, valor extensivo, supervivencia y profundización) de España por los componentes de Alemania. A través de los ejercicios contra-factuales podremos identificar qué componente o componentes explican las diferencias observadas entre ambos países en cuanto a crecimiento de las exportaciones se refiere, lo que en última instancia nos ayudará a identificar qué características del crecimiento exportador de Alemania permitirían a España crecer como lo ha hecho Alemania.

Para ilustrar el caso concreto de la contribución de la entrada al crecimiento de las exportaciones, la ecuación [2] se modifica de la siguiente manera:

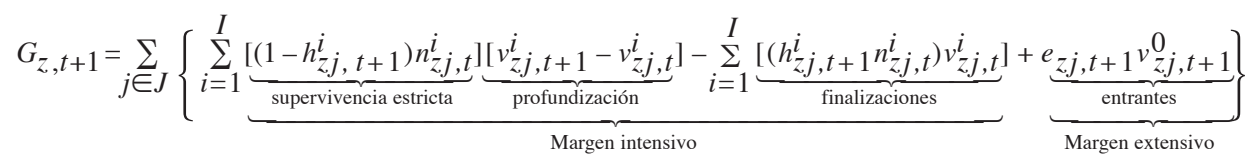


donde $« C F »$ denota el valor del contra-factual, en este caso $e_{z j, t+1}^{C F}$ es el número de nuevas relaciones comerciales en la industria $j$ perteneciente al sector tecnológico $z$ en el año $t+1$ en Alemania. Si queremos evaluar los efectos del valor extensivo, la ecuación pasa a ser,

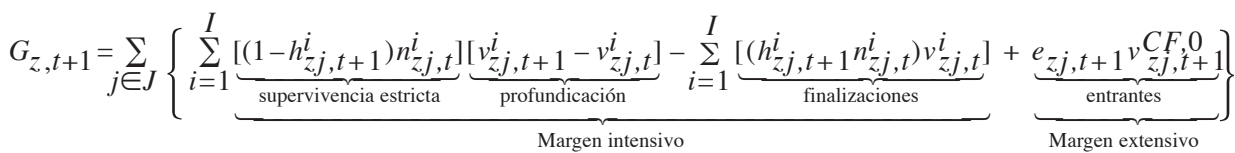

donde $v_{z, j+1}^{C F, 0}$ es el valor de comercio medio de cada una de las nuevas relaciones comerciales en la industria $j$ perteneciente al sector tecnológico $z$ en el año $t+1$ en Alemania.

Análogamente, los contra-factuales del componente de supervivencia y de profundización se calculan, respectivamente, como:

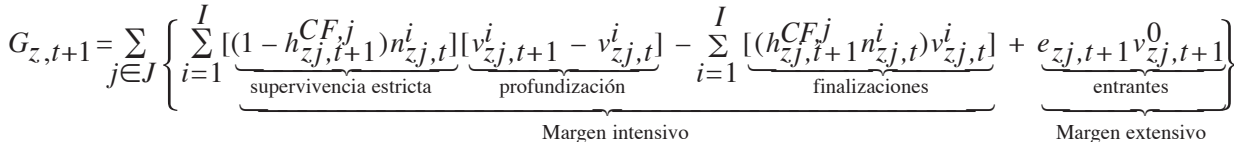

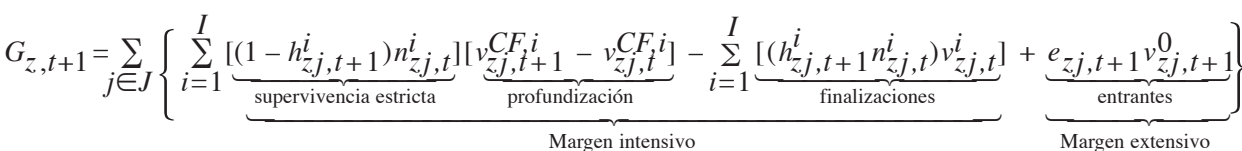

donde $h_{z j, t+1}^{C F, j}$ es la tasa de riesgo de terminación contra-factual de una relación comercial de la industria $j$ perteneciente al sector tecnológico $z$ con duración $i$ en el año $t+1$ de Alemania y $\left[v_{z j, t+1}^{C F, i}-v_{z j, t}^{C F, i}\right]$ es la profundización contra-factual (alemana) de la industria $j$ perteneciente al sector tecnológico $z$ con duración $i$ en el año $t+1$.

\subsection{Resultados}

Como hemos explicado en la sección anterior, utilizamos la ecuación [2] para realizar los ejercicios contra-factuales con el fin de analizar la contribución de cada uno de los cuatro componentes (entrada, valor extensivo, supervivencia y profundización) al crecimiento del valor de las exportaciones españolas en cada sector tecnológico ${ }^{5}$. La interpretación de los resultados de los ejercicios contra-factuales es

5 A la hora de llevar a la práctica los ejercicios contra-factuales las ecuaciones [1] y [2] han sido transformadas en sus correspondientes tasas de crecimiento de $t$ a $t+1$ tras dividir sus fórmulas por $V_{z, t}$ $=n_{z, t} v_{z, t}$, donde $V_{z, t}$ es el valor de las exportaciones totales en el año $t$ para el sector tecnológico $z$, y $n_{z, t}$ es el número de relaciones comerciales en $t$ para el sector tecnológico $z, \mathrm{y} v_{z, t}$ es el valor medio de 
como sigue: si para alguno de los componentes analizados individualmente existe un cambio importante en el crecimiento total de las exportaciones (tanto positivo como negativo), entonces podemos concluir que dicho componente es clave a la hora de explicar las diferencias en el crecimiento de las exportaciones entre España y Alemania. En el caso opuesto, cuando no hay diferencias significativas entre las tasas de crecimiento observado y contra-factual de España para el componente sustituido, podemos concluir que ese componente no es importante a la hora de explicar diferencias en el crecimiento de las exportaciones entre ambos países. Por tanto, utilizando los componentes del crecimiento de las exportaciones de Alemania como contra-factuales podemos evaluar si: uno, es el margen intensivo (supervivencia o profundización) o el margen extensivo (entrada o valor extensivo) el que, en última instancia, explica las diferencias en la evolución de las exportaciones españolas frente a las alemanas; y, dos, cuáles son los puntos débiles/fuertes del crecimiento de las exportaciones manufactureras en España.

El Cuadro 7 presenta los resultados de nuestro ejercicio de contra-factuales. En la primera columna del Cuadro 7 presentamos la tasa de crecimiento real de las exportaciones observada para cada uno de los cuatro sectores tecnológicos para España, en el periodo 1991-2005. Las siguientes columnas (de la 2 a la 5) muestran el diferencial respecto al crecimiento observado que hubieran experimentado las exportaciones españolas en caso de que los cuatro componentes, uno a uno, hubieran sido reemplazados por su valor en Alemania en lugar de su valor en España, manteniendo el resto de componentes en sus valores para España.

\section{CUADRO 7 \\ RESULTADOS DEL EJERCICIO CONTRA-FACTUAL (PAÍS DE REFERENCIA ALEMANIA). PERIODO DE ANÁLISIS: 1991-2005}

\begin{tabular}{|l|c|c|c|c|c|}
\hline \multicolumn{2}{|l|}{} & \multicolumn{3}{|c|}{$\begin{array}{c}\text { Diferencial respecto al crecimiento observado para España } \\
\text { utilizando Alemania como contra-factual }\end{array}$} \\
\hline & $\begin{array}{c}\text { Crecimiento } \\
\text { medio anual } \\
\text { observado (\%) }\end{array}$ & $\begin{array}{c}\text { Riesgo } \\
\mathbf{( \% )}\end{array}$ & $\begin{array}{c}\text { Profundización } \\
\mathbf{( \% )}\end{array}$ & $\begin{array}{c}\text { Entrada } \\
\mathbf{( \% )}\end{array}$ & $\begin{array}{c}\text { Valor } \\
\text { extensivo }\end{array}$ \\
\hline España & & & & & \\
Bajo & 4,58 & 1,49 & $-2,49$ & $-0,64$ & $-0,65$ \\
Medio-bajo & 4,18 & 2,32 & $-0,48$ & $-1,89$ & $-2,43$ \\
Medio-alto & 4,85 & 2,45 & $-1,95$ & $-0,66$ & $-1,08$ \\
Alto & 6,17 & 2,89 & 0,19 & $-2,98$ & $-2,48$ \\
\hline
\end{tabular}

las exportaciones de cada relación comercial en el año $t$ para el sector tecnológico $z$. Tras esta transformación, que habría complicado innecesariamente los apartados metodológicos en este trabajo, los distintos elementos en los que se descompone la tasa de crecimiento de las exportaciones tienen la interpretación, y han sido utilizados, en porcentajes. 
En el sector de tecnología baja, el componente de riesgo alemán hubiera tenido un efecto positivo sobre el crecimiento de las exportaciones españolas para dicho sector. El aumento de las exportaciones hubiera sido del 1,49 por 100, lo que habría representado un crecimiento contra-factual anual de las exportaciones del 6,07 por 100 , frente al observado de 4,58 por 100 . Si este sector hubiera tenido el componente alemán de profundización, las exportaciones hubieran sido significativamente menores, ya que este componente habría tenido un efecto negativo de -2,49 por 100 . El efecto de este componente hubiera provocado que las exportaciones contra-factuales hubieran crecido sólo un 2,10 por 100 frente al 4,58 por 100 observado. Por último, los dos componentes alemanes del margen extensivo también hubieran tenido un impacto negativo, aunque menor que en el caso de la profundización $(-0,64$ por 100 para la entrada y $-0,65$ por 100 para el valor extensivo, lo que hubiera provocado un crecimiento contra-factual conjunto inferior y del 3,29 por 100 , frente al 4,58 por 100 observado). Por tanto, el fuerte crecimiento de las exportaciones españolas en el sector de tecnología baja se podría atribuir principalmente a la buena evolución del componente de profundización español.

Los resultados para el sector de tecnología media-baja son similares al sector anterior en cuanto al signo de los componentes, pero difieren en la importancia relativa de los mismos. De nuevo, el componente de riesgo alemán volvería a tener un impacto significativo y positivo sobre el crecimiento potencial de las exportaciones españolas para dicho sector. El crecimiento de las exportaciones españolas hubiera sido de 6,50 por 100 en lugar de 4,18 por 100 si la tasa de riesgo de España hubiera sido la de Alemania. Sin embargo, son los componentes del margen extensivo los que mejor explican el diferencial de crecimiento de las exportaciones españolas respecto a las alemanas. Así, el crecimiento de las exportaciones españolas hubiera sido aproximadamente la mitad si las tasas de entrada o de valor extensivo en España hubieran sido las de Alemania (2,29 por 100 y 1,75 por 100 , respectivamente, en lugar de 4,18 por 100$)$.

En el sector de tecnología media-alta, el componente de supervivencia de Alemania volvería a mostrar un impacto positivo y elevado sobre el crecimiento potencial de las exportaciones españolas para dicho sector. Sin embargo, el componente de profundización de Alemania hubiera tenido un efecto significativo y negativo. En relación al crecimiento medio anual observado (4,85 por 100) las exportaciones españolas hubieran crecido un 7,30 por 100 si la tasa de riesgo española hubiera sido la de Alemania, pero sólo un 2,90 por 100 si la tasa de profundización de España hubiera sido la de Alemania. En relación con el margen extensivo, de nuevo los componentes alemanes de este margen hubieran tenido un efecto negativo en las exportaciones españolas, aunque significativamente menor que el de la profundización. Así, si España hubiera tenido las tasas de entrada o de valor extensivo de Alemania sus exportaciones hubieran crecido un 4,19 por 100 y un 3,77 por 100 , respectivamente, en lugar de un 4,85 por 100 .

Por último, analizamos los resultados para el contra-factual del sector de tecnología alta. En este sector la tasa de riesgo alemana habría sido la que más hubiera contribuido a aumentar las exportaciones españolas, que habrían tenido un creci- 
miento potencial de 9,06 por 100 en lugar de 6,17 por 100 . En cuando a los otros componentes, si España hubiera tenido el componente de profundización de Alemania el crecimiento de las exportaciones españolas también hubiera sido superior al observado, aunque la magnitud del cambio hubiera sido muy pequeña (6,36 por 100 frente al 6,17 por 100 observado). Por último, los dos componentes alemanes del margen extensivo son los que hubieran reducido significativamente el crecimiento de las exportaciones españolas. Las exportaciones españolas hubieran crecido sólo un 3,19 por 100 ó un 3,69 por 100 si la tasa de entrada o el valor extensivo, respectivamente, hubieran sido en España como en Alemania, frente al crecimiento observado del 6,17 por 100 .

\section{Conclusiones}

En este trabajo hemos analizado el crecimiento de las exportaciones de manufacturas españolas y alemanas por sectores de contenido tecnológico (bajo, medio-bajo, medio-alto y alto) a través de los márgenes intensivo y extensivo de comercio, para el periodo 1991-2005, utilizando datos de exportaciones bilaterales de productos manufacturados obtenidos de Naciones Unidas (COMTRADE). Estos márgenes se pueden descomponer en varios componentes: la creación de nuevas relaciones comerciales, el valor promedio de las nuevas relaciones comerciales, el grado de persistencia o vida media de las relaciones comerciales existentes, y el valor promedio de las relaciones comerciales que sobreviven un año tras otro. Para estudiar el impacto de cada uno de estos componentes, primero, los analizamos de un modo descriptivo, tanto para España como para Alemania, buscando similitudes y diferencias en la evolución de las exportaciones de estos dos países y analizando el papel de cada uno de los componentes. A continuación, hemos llevado a cabo un análisis contra-factual similar al propuesto por Besedes y Prusa (2011) con el fin de identificar los componentes fuertes y débiles de las exportaciones españolas con respecto a las de Alemania.

Nuestros resultados del ejercicio de contra-factuales indican que el componente de supervivencia habría sido el elemento más «débil» en el crecimiento de las exportaciones españolas en los cuatro sectores tecnológicos, siendo su importancia creciente con la intensidad tecnológica del sector analizado. Sin embargo, los dos componentes del margen extensivo (la tasa de entrada y el valor extensivo) habrían sido, en general, los elementos «fuertes» de las exportaciones españolas, si bien hay un componente del margen intensivo (la profundización en valor de las relaciones comerciales que sobreviven) que ha sido especialmente relevante para los sectores de tecnología baja y media-alta. Es importante resaltar que estos resultados son diferentes de los que han obtenido estudios previos como Felbermayr y Kohler (2006), Helpman et al. (2008) y Besedeš y Prusa (2011), quienes encuentran que el crecimiento del comercio viene explicado en su mayoría por el margen intensivo, pero están en línea con los resultados de Bernard et al. (2009) para EEUU y de De Lucio et al. (2011) para España, quienes, utilizando una metodología diferente, encuentran que para un periodo largo de 
tiempo, el crecimiento total de las exportaciones de un país estaría explicado a partes iguales por el margen intensivo y el margen extensivo.

Desde el punto de vista de las posibles recomendaciones de política económica, y dado que el componente de supervivencia se revela como el más importante en el ejercicio de contra-factuales, convendría realizar un esfuerzo para mejorar la supervivencia de las relaciones comerciales españolas. De ese modo, todos los sectores tecnológicos podrían aumentar el crecimiento de sus exportaciones (y en especial el sector de contenido tecnológico alto). Todo ello, sin olvidar la importancia del mantenimiento y apoyo a los componentes del margen extensivo, esto es, a la continuidad de unas tasas de entrada en la actividad exportadora y de valor extensivo de las nuevas relaciones comerciales que sean al menos como las observadas en el periodo 1991-2005 (y, a ser posible, con la fortaleza mostrada por los sectores de contenido tecnológico medio-bajo y alto).

\section{Referencias bibliográficas}

[1] AMURGO-PACHECO, A. y PIEROLA, M. D. (2007): «Patterns of export diversification in developing countries: intensive and extensive margins», HEI Working Paper n. ${ }^{\circ} 20 / 2007$.

[2] BERNARD, A.B.; JENSEN, J.B.; REDDING, S.J. y SCHOTT, P.K. (2009): «The margins of US trade», American Economic Review: Papers \& Proceedings, 99, 2, 487-493.

[3] BESEDES̆, T. y PRUSA, T.J. (2011): «The role of extensive and intensive margins and export growth», Journal of Development Economics, 96, 371-379.

[4] BRENTON, P. y NEWFARMER, R. (2007): «Watching more than the discovery channel: Export cycles and diversification in development», World Bank Policy Research Working Paper n. ${ }^{\circ} 4302$.

[5] DE LUCIO, J.; MÍNGUEZ-FUENTES, R.; MINONDO, A. y REQUENA-SILVENTE, F. (2011): «The extensive and intensive margins of Spanish trade», International Review of Applied Economics, 1-17.

[6] EATON, J.; ESLAVA, M.; KLUGER, M. y TYBOUT, J. (2007): «Export dynamics in Colombia: Firm-level evidence», NBER Working Paper, n. ${ }^{\circ} 13531$.

[7] EVENETT, S. J. y VENABLES, A.J. (2002): «Export growth in developing countries: Market entry and bilateral trade flows», University of Bern Working Paper, mimeo.

[8] FELBERMAYR, G.J. y KOHLER, W. (2006): «Exploring the intensive and extensive margins of world trade», Review of World Economics, 142(4), 642-674.

[9] HELPMAN, E.; MELITZ, M. y RUBINSTEIN, Y. (2008): «Estimating trade flows: Trading partners and trading volumes», Quarterly Journal of Economics, 123(2), 441487.

[10] HUMMELS, D. y KLENOW, P.L. (2005): «The variety and quality of a nation's exports», American Economic Review, 95(3), 704-723.

[11] KANG, K. (2004): «The path of the extensive margin (export variety), theory and evidence», University of California, Davis Working Paper, mimeo.

[12] MINONDO, A. y REQUENA, F. (2010): «The intensive and extensive margins of trade decomposing exports growth differences across Spanish regions», FUNCAS Documento de Trabajo 525/2010. 
APÉNDICE

\section{CLASIFICACIÓN DE INDUSTRIAS CUCI POR CONTENIDO TECNOLÓGICO}

\begin{tabular}{|l|l|}
\hline $\begin{array}{c}\text { Sector de contenido } \\
\text { tecnológico }\end{array}$ & \multicolumn{1}{c|}{ Industria } \\
\hline Bajo & $\begin{array}{l}\text { Manufacturas del productos de alimentación, bebida y tabaco } \\
\text { Manufacturas del textil y sus productos } \\
\text { Manufacturas de la piel y sus productos } \\
\text { Manufacturas de la madera y sus productos } \\
\text { Manufacturas del papel y sus productos; imprenta y edición } \\
\text { Manufacturas (las demás), excl. reciclaje }\end{array}$ \\
\hline Medio-bajo & $\begin{array}{l}\text { Manufacturas del carbón, petróleo y sus productos y combustible nuclear } \\
\text { Manufacturas del caucho y del plástico } \\
\text { Manufacturas del otros productos minerales no metálicos } \\
\text { Manufacturas del metales básicos } \\
\text { Manufacturas de productos de metal, excepto maquinaria y equipamiento } \\
\text { Manufacturas del transporte marítimo y su reparación }\end{array}$ \\
\hline Medio-alto & $\begin{array}{l}\text { Manufacturas de maquinaria y equipamiento mecánico } \\
\text { Manufacturas de maquinaria y aparatos eléctricos } \\
\text { Manufacturas de transporte de vehículos a motor, trailers y semi-trailers } \\
\text { Manufacturas del transporte ferroviario } \\
\text { Manufacturas de motocicletas y bicicletas } \\
\text { Manufacturas de otros modos de transporte } \\
\text { Manufacturas de la química básica } \\
\text { Manufacturas de pesticidas y otros productos agro-químicos } \\
\text { Manufacturas de pinturas, barnices, tintas y similares } \\
\text { Manufacturas de detergentes, productos de limpieza, perfumes y productos } \\
\text { de belleza } \\
\text { Manufacturas de otros productos químicos } \\
\text { Manufacturas de fibras artificiales }\end{array}$ \\
\hline $\begin{array}{l}\text { Manufacturas del maquinaria de oficina y ordenadores } \\
\text { Manufacturas del aparatos de radio, televisión y comunicación } \\
\text { Manufacturas de equipamiento medico, de medición y precisión, óptica, relojes } \\
\text { Industria de aviación y aeroespacial } \\
\text { Industria farmacéutica }\end{array}$ \\
\hline
\end{tabular}

FUENTE: OCDE 